\title{
Two-centered magical charge orbits
}

\author{
Laura Andrianopoli, ${ }^{a, b}$ Riccardo D’Auria, ${ }^{a, b}$ Sergio Ferrara, ${ }^{c, d, e}$ Alessio Marrani $^{c}$ \\ and Mario Trigiante ${ }^{a, b}$ \\ ${ }^{a}$ Dipartimento di Fisica, Politecnico di Torino, \\ Corso Duca degli Abruzzi 24, I-10129, Italy \\ ${ }^{b}$ INFN - Sezione di Torino, \\ Via Pietro Giuria, 1 I-10125 Torino, Italy \\ ${ }^{c}$ Physics Department, Theory Unit, CERN, \\ CH -1211, Geneva 23, Switzerland \\ ${ }^{d}$ INFN - Laboratori Nazionali di Frascati, \\ Via Enrico Fermi 40, I-00044 Frascati, Italy \\ ${ }^{e}$ Department of Physics and Astronomy, University of California, \\ Los Angeles, CA 90095-1547, U.S.A. \\ E-mail: laura.andrianopoli@polito.it, riccardo.dauria@polito.it, \\ mario.trigiante@polito.it, sergio.ferrara@cern.ch, \\ alessio.marrani@cern.ch
}

AbStRaCt: We determine the two-centered generic charge orbits of magical $\mathcal{N}=2$ and maximal $\mathcal{N}=8$ supergravity theories in four dimensions. These orbits are classified by seven $U$-duality invariant polynomials, which group together into four invariants under the horizontal symmetry group SL $(2, \mathbb{R})$. These latter are expected to disentangle different physical properties of the two-centered black-hole system. The invariant with the lowest degree in charges is the symplectic product $\left\langle\mathcal{Q}_{1}, \mathcal{Q}_{2}\right\rangle$, known to control the mutual nonlocality of the two centers.

Keywords: Black Holes, Supergravity Models

ArXiv EPrint: 1101.3496 


\section{Contents}

1 Introduction 1

2 Little group of $p$ charge vectors in irreducible symmetric models 3

$2.1 J_{3}^{\mathbb{Q}}(\mathcal{N}=2), J_{3}^{\mathbb{Q}_{s}}(\mathcal{N}=8) \quad 4$

$2.2 J_{3}^{\mathbb{H I}}(\mathcal{N}=2 \leftrightarrow \mathcal{N}=6) \quad 6$

$2.3 J_{3}^{\mathbb{C}}(\mathcal{N}=2), M_{1,2}(\mathbb{O})(\mathcal{N}=5) \quad 7$

$2.4 J_{3}^{\mathbb{R}}(\mathcal{N}=2) \quad 9$

3 Invariant structures and the role of the horizontal symmetry $\mathrm{SL}_{h}(2, \mathbb{R}) \quad 11$

4 Two-centered orbits with non-compact stabiliser: the $\mathcal{N}=8$ BPS and $\begin{array}{ll}\text { octonionic } \mathcal{N}=2 \text { non-BPS cases } & 15\end{array}$

\section{Introduction}

Multi-centered black-hole solutions of supergravity theories in $d=4$ space-time dimensions have recently received much attention, especially in connection to the classification of nonperturbative string BPS states and their brane interpretation [1-3]. A generalisation of the attractor Mechanism [4-8] (for a review, see e.g. [9]) has been shown to occur, as firstly pointed out by Denef [10], called split attractor flow for BPS $\mathcal{N}=2$ black holes $[1-3,10-12]$.

Attempts to generally classify the two-centered solutions of supergravity theories with symmetric scalar manifolds and electric-magnetic duality $\left(U\right.$-duality $\left.{ }^{1}\right)$ symmetry given by classical Lie groups have been considered [16-18]. In particular, within the framework of the minimal coupling [19] of vector multiplets to $\mathcal{N}=2$ supergravity, it was shown in [17] that different physical properties, such as marginal stability and split attractor flow solutions, can be classified by duality-invariant constraints, which in this case involve two dyonic black-hole charge vectors, and not only one.

This leads one to consider the mathematical issue of the classification of orbits of two (or more) dyonic charge vectors in the context of multi-centered black-hole physics. For the theories treated in $[17,18]$, the charge vector lies in the fundamental representation of $U(1, n)$ (minimally coupled $\mathcal{N}=2$ supergravity [19]) and in the spinor-vector representation of $\mathrm{SL}(2, \mathbb{R}) \times \mathrm{SO}(q, n)$, corresponding to reducible cubic $\mathcal{N}=2$ sequence [20-22] for $q=2$, and to matter-coupled $\mathcal{N}=4$ supergravity for $q=6$.

In [17], the two-centered $U$-invariant polynomials of the minimally coupled theory were constructed, and shown to be four (dimension of the adjoint of the two-centered horizontal symmetry $U(2))$. The same was done for the aforementioned cubic sequence in [18], where

\footnotetext{
${ }^{1}$ Here $U$-duality is referred to as the "continuous" symmetries of $[13,14]$. Their discrete versions are the $U$-duality non-perturbative string theory symmetries introduced by Hull and Townsend [15].
} 
the number of $U$-invariants were computed to be seven for $n \geqslant 2$, six for $n=1$ and five for the irreducible $t^{3}$ model.

It is the aim of the present investigation to generalise these results to four-dimensional supergravity theories with symmetric irreducible scalar manifolds, in particular to the $\mathcal{N}=8$ maximal theory and to the $\mathcal{N}=2$ magical models.

We find that when the stabilizer of a two-centered charge orbit is non-compact, the corresponding orbit is not unique. As we will consider in section 4, this feature is also exhibited by the classification of the orbits of two non-lightlike vectors in a pseudo-Euclidean space $E_{p, q}$ of dimension $p+q$ and signature $(p, q)$. A prominent role is played by an emergent horizontal symmetry $\mathrm{SL}_{h}(2, \mathbb{R})$, whose invariants classify all possible two-vector orbits.

In this respect, the aforementioned $t^{3}$ model, whose $U$-duality group is SL $(2, \mathbb{R})$, provides a simple yet interesting example, because it may be obtained both as rank- 1 truncation of the reducible symmetric models and as first, non-generic element of the sequence of irreducible $\mathcal{N}=2$ symmetric models, which contains the four rank-3 magical supergravity theories mentioned above. The two-centered configurations and the generic (BPS) orbit $\mathcal{O}=\mathrm{SL}(2, \mathbb{R})$ of $t^{3}$ model were studied in section 7 of [18], in which it was pointed out that, as it occurs also for the one-centered case [23], no stabilizer for the two-centered orbit exists. $^{2}$ The five components of the spin $s=2$ horizontal tensor $\mathbf{I}_{a b c d}$ (defined in (3.12) below, and explicitly given by (3.15)-(3.19)) form a complete basis of duality-invariant polynomials [18]; as a consequence, the counting (2.2) for $p=2$-centered black hole solutions in the $t^{3}$ model simply reads $5+3-0=4 \times 2$, because $I_{p=2}=5$ and $\operatorname{dim}_{\mathbb{R}}\left(\mathcal{G}_{p}\right)=0$. Moreover, there exist only two independent $\left[\mathrm{SL}_{h}(2, \mathbb{R}) \times \mathrm{SL}(2, \mathbb{R})\right]$-invariant polynomials, which can be taken to be the symplectic product $\mathcal{W}$ (of order two in charges, defined in (3.9) below) and $\mathbf{I}_{6}$ (of order six in charges, defined in (3.24) below); an alternative choice of basis for the SL $(2, \mathbb{R})$-invariant polynomials is thus e.g. given by three components of $\mathbf{I}_{a b c d}$ out of the five (3.15)-(3.19), and the two horizontal invariants $\mathcal{W}$ and $\mathbf{I}_{6}$.

The plan of the paper is as follows.

In section 2 we give a group theoretical method (based on progressive branchings of symmetry groups, considered as complex groups) to find the multi-centered charge orbits of a theory with a symmetric scalar manifold; we then apply it to all irreducible symmetric cases. The analysis of this section will not depend on the real form of the stabilizer of the orbit, and the results will then hold both for BPS and all the non-BPS orbits of the given model. In section 3 we propose a complete basis for $U$-duality polynomials in the presence of two dyonic black-hole charge vectors in irreducible symmetric models, and we also consider the role of the horizontal symmetry in this framework. section 4 extends the analysis of section 2 to different non-compact real forms of the stabilizer of one-centered charge orbits related to Jordan algebras over the octonions, namely to $\mathcal{N}=8$ theory (whose $\frac{1}{8}$-BPS one-centered stabilizer is $E_{6(2)}$ ) and for exceptional magical $\mathcal{N}=2$ theory (whose BPS and non-BPS $\mathcal{I}_{4}>0$ one-centered stabilizers are the compact $E_{6(-78)}$ and the non-compact $E_{6(-14)}$, respectively).

Possible extensions of the present investigation may also cover composite configurations

\footnotetext{
${ }^{2}$ As it holds for the magical $J_{3}^{\mathbb{R}}$ model, see table I.
} 
with "small" constituents, as well as a detailed study of the multi-centered charge orbits in $\mathcal{N}=5,6$-extended supergravity theories.

\section{Little group of $p$ charge vectors in irreducible symmetric models}

We consider a $p$-center black hole solution in a Maxwell-Einstein supergravity theory in $d=4$ space-time dimensions.

The $p$ dyonic black-hole charge vectors can be arranged as

$$
\mathbf{Q}_{a} \equiv\left\{\mathcal{Q}_{a}^{M}\right\}_{M=1, \ldots, f},
$$

where $\mathcal{Q}_{a}^{M}$ sits in the irreducible representation $\left(\mathbf{p}, \operatorname{Sympl}\left(G_{4}\right)\right)$ of the group $\operatorname{SL}_{h}(p, \mathbb{R}) \times$ $G_{4}$. $\mathbf{p}$ is the fundamental representation (spanned by the index $a=1, \ldots, p$ ) of the horizontal symmetry group [18] $\mathrm{SL}_{h}(p, \mathbb{R})$ (see section 3), while $\mathbf{S y m p l}\left(G_{4}\right)$ is the symplectic irreducible representation of the black-hole charges, spanned by the index $M=1, \ldots, f$ of the $U$-duality group $G_{4}$, where $f \equiv \operatorname{dim}_{\mathbb{R}}\left(\operatorname{Sympl}\left(G_{4}\right)\right)$.

Suppose there are $I_{p}$ independent $G_{4}$-invariant polynomials constructed out of $\mathbf{Q}_{a}$, and let $\mathcal{G}_{p}$ denote the little group of the system of charges, defined as the largest subgroup of $G_{4}$ such that $\mathcal{G}_{p} \mathbf{Q}_{a}=\mathbf{Q}_{a} \forall a$. Then, the following relation ${ }^{3}$ holds [18]:

$$
I_{p}+\operatorname{dim}_{\mathbb{R}}\left(G_{4}\right)-\operatorname{dim}_{\mathbb{R}}\left(\mathcal{G}_{p}\right)=f p .
$$

Some preliminary general observations are in order:

- The group theoretical analysis of the present section does not depend on the real form of $G_{4}$ and $\mathcal{G}_{p}$. We will then generally consider the complex groups. From a physical point of view, the BPS and non-BPS cases in various supergravity theories correspond to different choices of non-compact real forms of $\mathcal{G}_{p}$ (and of $G_{4}$, as well). However, for BPS orbits in $\mathcal{N}=2$ symmetric models, and in particular for magical models, the stabilizer is always the compact form of the relevant group (see table 1).

- We shall generally assume $\mathbf{Q}_{1}$ to be in a representation corresponding to a "large" black hole, ${ }^{4}$ namely such that the quartic invariant $\mathcal{I}_{4}\left(\mathcal{Q}_{1}^{4}\right) \neq 0$.

- We shall consider "generic" orbits, in which all $I_{p}$ invariants are independent.

- There are two relevant cases, corresponding to different behaviors in the counting of invariants:

a) The largest subgroup commuting with $\mathcal{G}_{p}$ inside $G_{4}$ is $\mathrm{U}(1) \subset G_{4}$, so that $\mathcal{G}_{p} \times$ $\mathrm{U}(1) \subset G$.

b) $\mathrm{A} \mathrm{U}(1)$ commuting with $\mathcal{G}_{p}$ inside $G_{4}$ does not exist.

\footnotetext{
${ }^{3} \mathrm{~A}$ necessary but not sufficient condition for eq. (2.2) to hold is $p<f$, such that the $p$ dyonic charge vectors can all be taken to be linearly independent.

${ }^{4}$ Multi-center configurations with "small" constituents [12, 24, 25] can be treated as well, and they will be considered elsewhere.
} 


\begin{tabular}{|c|c|}
\hline$J_{3}^{\mathbb{A}}$ & $\mathcal{O}_{p=2, B P S}=\frac{\operatorname{Conf}\left(J_{3}^{\mathrm{A}}\right)}{\mathcal{G}_{p=2}\left(J_{3}^{\mathrm{A}}\right)}$ \\
\hline$J_{3}^{\mathbb{Q}}$ & $\frac{E_{7(-25)}}{\mathrm{SO}(8)}$ \\
\hline$J_{3}^{\mathbb{H}}$ & $\frac{\mathrm{SO} *(12)}{[S U(2)]^{3}}$ \\
\hline$J_{3}^{\mathbb{C}}$ & $\frac{S U(3,3)}{[\mathrm{U}(1)]^{2}}$ \\
\hline$J_{3}^{\mathbb{R}}$ & $S p(6, \mathbb{R})$ \\
\hline
\end{tabular}

Table 1. BPS generic charge orbits of 2-centered extremal black holes in $\mathcal{N}=2, d=4$ magical models. $\operatorname{Conf}\left(J_{3}^{\mathbb{A}}\right)$ denotes the "conformal" group of $J_{3}^{\mathbb{A}}$ (see e.g. [26], and refs. therein). By introducing $\mathbb{A}=\mathbb{R}, \mathbb{C}, \mathbb{H}, \mathbb{O}$, it is worth remarking that the stabilizer group $\mathcal{G}_{p=2}\left(J_{3}^{\mathbb{A}}\right)$ and the automorphism group $A u t\left(\mathbf{t}(\mathbb{A})\right.$ ) of the normed triality $\mathbf{t}(\mathbb{A})$ in $\operatorname{dimension} \operatorname{dim}_{\mathbb{R}} \mathbb{A}=1,2,4,8$ (given e.g. in eq. (5) of [27]) share the same Lie algebra. In other words, $\mathfrak{g}_{p=2}\left(J_{3}^{\mathbb{A}}\right) \sim \operatorname{tri}(\mathbb{A})$, where $\mathfrak{t r i}(\mathbb{A})$ denotes the Lie algebra of $A u t(\mathbf{t}(\mathbb{A}))$ itself (see e.g. eq. (21) of [27]).

In the case $b$ ), all the singlets in the decomposition of $G_{4} \rightarrow \mathcal{G}_{p}$ correspond to $p$-center $G_{4}$-invariant polynomials of $\operatorname{Sympl}\left(G_{4}\right)$. On the other hand, in the case $a$ ) the number of singlets corresponds to the number of $p$-center $G_{4}$-invariant polynomials, plus one if some of them are charged with respect to $\mathrm{U}(1)$, because one of the singlets can still be acted on by the corresponding $\mathrm{U}(1)$-grading.

- The general method for working out $\mathcal{G}_{p}$ and thus $I_{p}$, having solved the problem for $p-1$ centers, is to consider the $p^{\text {th }}$ charge vector $\mathbf{Q}_{p}$ as transforming in a (reducible) representation of the little group $\mathcal{G}_{p-1}$ of the former $p-1$ charges, and solve the corresponding one-charge-vector problem.

In the next subsections we will consider the cases $p=1$ and $p=2$ in all irreducible symmetric cases pertaining to supergravity theories in $d=4$ dimensions (with the exception of the rank-1 $t^{3}$ model, treated in [18]). In the case $p=1$, we will retrieve the well known result $I_{p=1}=1$, whereas in the $p=2$ case we will obtain $I_{p=2}=7$ in all cases under consideration.

\section{$2.1 J_{3}^{\mathbb{Q}}(\mathcal{N}=2), J_{3}^{\mathbb{O}_{s}}(\mathcal{N}=8)$}

Let us start considering the exceptional case, based on the Euclidean degree-3 Jordan algebra $J_{3}^{\mathbb{Q}}$ on the octonions $\mathbb{O}$. Since, as mentioned earlier, we actually work with complex groups, this case pertains also to maximal $\mathcal{N}=8$ supergravity, based on the Euclidean degree-3 Jordan algebra $J_{3}^{\mathbb{Q}_{s}}$ on the split octonions $\mathbb{O}_{s}$.

In the complex field, $G_{4}=E_{7}$ and $\operatorname{Sympl}\left(E_{7}\right)=$ Fund $\left(E_{7}\right)=\mathbf{5 6}$.

- Let us first solve the one-center problem $(p=1)$. $\mathcal{G}_{1}$ is a real form of $E_{6}$; the $\mathbf{5 6}$ branches with respect to $E_{6}$ as follows (subscripts denote the $U(1)$-charges through- 
out):

$$
\mathbf{5 6} \rightarrow \mathbf{1}_{-3}+\mathbf{2} \mathbf{7}_{-1}+\overline{\mathbf{2 7}}_{+1}+\mathbf{1}_{+3},
$$

and correspondingly the charge vector $\mathbf{Q}_{1}$ (defined as $\left(p^{\Lambda}, q_{\Lambda}\right)$ throughout) decomposes as follows:

$$
\mathbf{Q}_{1}=\left(p^{0}, \mathbf{p}_{\mathbf{2 7}}, q_{0}, \mathbf{q}_{\overline{\mathbf{2 7}}}\right) .
$$

Note that the branching (2.3) contains two $E_{6}$-singlets, and $E_{7} \supset E_{6} \times \mathrm{U}(1)=$ $\mathcal{G}_{1} \times \mathrm{U}(1)$. According to the previous discussion, one of the singlets can be freely acted on by the $\mathrm{U}(1)$. Thus, by acting with $G_{4} / \mathcal{G}_{1}=E_{7} / E_{6}$, the 1-center charge vector $\mathbf{Q}_{1}$ can be reduced as follows:

$$
\mathbf{Q}_{1} \stackrel{E_{7} / E_{6}}{\longrightarrow}\left(I^{(1)}, \mathbf{0}_{\mathbf{2 7}}, \pm I^{(1)}, \mathbf{0}_{\overline{\mathbf{2 7}}}\right) .
$$

One is then left with only one independent singlet charge $I^{(1)}$ related to the 1-center quartic invariant $\mathcal{I}_{4}\left(\mathcal{Q}_{1}^{4}\right)$; therefore, $I_{1}=1$, as expected. This analysis is consistent with the general formula (2.2), which in this case reads:

$$
I_{1}+\operatorname{dim}_{\mathbb{R}}\left(E_{7}\right)-\operatorname{dim}_{\mathbb{R}}\left(E_{6}\right)=1+133-78=56 .
$$

- Let us now proceed to deal with the two charge-vector problem $(p=2)$. The second charge vector is denoted as $\mathbf{Q}_{2} \equiv\left(m^{\Lambda}, e_{\Lambda}\right)$ throughout. Having solved the problem for $p=1$, we can decompose $\mathbf{Q}_{2}$ with respect to $\mathcal{G}_{1}=E_{6}$ using (2.3), obtaining the decomposition

$$
\mathbf{Q}_{2}=\left(I^{(2)}, \mathbf{m}_{\mathbf{2 7}}, I^{(3)}, \mathbf{e}_{\overline{\mathbf{2 7}}}\right),
$$

and then determine the corresponding little group inside $E_{6}$. The little group of the irreducible representation $\mathbf{2 7}$ of $E_{6}$ is $F_{4}$, under which

$$
27 \rightarrow 1+26,
$$

and correspondingly

$$
\mathbf{m}_{\mathbf{2 7}} \rightarrow\left(I^{(4)}, \mathbf{m}_{\mathbf{2 6}}\right) ; \quad \mathbf{e}_{\overline{\mathbf{2 7}}} \rightarrow\left(I^{(5)}, \mathbf{e}_{\mathbf{2 6}}\right) .
$$

Note in particular that $F_{4}$ is a maximal (symmetric) subgroup of $E_{6}$, so that all singlets correspond to extra $E_{7}$-invariant polynomials, and that $\mathbf{m}_{\mathbf{2 6}}$ can be set to zero through the action of $\mathcal{G}_{1} / F_{4}=E_{6} / F_{4}$, thus yielding the result:

$$
\mathbf{Q}_{2} \stackrel{E_{6} / F_{4}}{\longrightarrow}\left(I^{(2)}, I^{(4)}, \mathbf{0}_{\mathbf{2 6}}, I^{(3)}, I^{(5)}, \mathbf{e}_{\mathbf{2 6}}\right) \text {. }
$$

- The 26 of $F_{4}$ has little group $\mathrm{SO}(8)$, which does not commute with a U(1) in $F_{4}$. Under this non-maximal embedding, the $\mathbf{2 6}$ branches as

$$
26 \rightarrow 1+1+8_{\mathrm{v}}+8_{\mathrm{s}}+8_{\mathrm{c}}
$$

and correspondingly

$$
\mathbf{e}_{\mathbf{2 6}} \rightarrow\left(I^{(6)}, I^{(7)}, \mathbf{e}_{\mathbf{8}_{\mathbf{v}}}, \mathbf{e}_{\mathbf{8}_{\mathbf{s}}}, \mathbf{e}_{\mathbf{8}_{\mathrm{c}}}\right) .
$$

Therefore, by acting with $F_{4} / \mathcal{G}_{2}=F_{4} / \mathrm{SO}(8), \mathrm{Q}_{2}$ can then be put in the form

$$
\mathbf{Q}_{2} \stackrel{F_{4} / \mathrm{SO}(8)}{\longrightarrow}\left(I^{(2)}, I^{(4)}, \mathbf{0}_{\mathbf{2 6}}, I^{(3)}, I^{(5)}, I^{(6)}, I^{(7)}, \mathbf{0}_{\mathbf{8}_{\mathbf{v}}}, \mathbf{0}_{\mathbf{8}_{\mathbf{s}}}, \mathbf{0}_{\mathbf{8}_{\mathbf{c}}}\right) \text {. }
$$


In conclusion, we found that the little group of a 2-centered black-hole solution is $\mathcal{G}_{2}=\mathrm{SO}(8)$, and the corresponding 2-centered charge orbits correspond to different real forms of the quotient of complex groups

$$
\mathcal{O}_{p=2}=\frac{G_{4}}{\mathcal{G}_{2}}=\frac{E_{7}}{\mathrm{SO}(8)} .
$$

The $E_{7}$-invariant polynomials for a 2-centered configuration are seven: $I_{2}=7$; indeed, the general formula (2.2) gives:

$$
I_{2}+\operatorname{dim}_{\mathbb{R}}\left(E_{7}\right)-\operatorname{dim}_{\mathbb{R}}(\operatorname{SO}(8))=7+133-28=112=2 \cdot 56 .
$$

$\mathbf{2 . 2} J_{3}^{\mathbb{H}}(\mathcal{N}=2 \leftrightarrow \mathcal{N}=6)$

This model is based on the Euclidean degree-3 Jordan algebra $J_{3}^{\mathbb{H}}$ on the quaternions $\mathbb{H}$, and it is "dual" to $\mathcal{N}=6$ "pure" theory, because these theories share the same bosonic sector $[20,21,28-31]$.

In the complex field $G_{4}=\mathrm{SO}(12)$, and $\operatorname{Sympl}(\mathrm{SO}(12))=\mathbf{3 2}$, the chiral spinor irreducible representation of $\mathrm{SO}(12)$.

- Let us first solve the problem for $p=1$. $\mathcal{G}_{1}$ is a real form of $\mathrm{SU}(6)$, the relevant (maximal symmetric) embedding is

$$
\mathrm{SO}(12) \supset \mathrm{SU}(6) \times \mathrm{U}(1)=\mathcal{G}_{1} \times \mathrm{U}(1)
$$

and the $\mathbf{3 2}$ accordingly branches

$$
32 \rightarrow \mathbf{1}_{-3}+\mathbf{1 5}_{-1}+\overline{\mathbf{1 5}}_{+1}+\mathbf{1}_{+3},
$$

corresponding to the charge decomposition

$$
\mathbf{Q}_{1}=\left(p^{0}, \mathbf{p}_{15}, q_{0}, \mathbf{q}_{15}\right) .
$$

The analysis here is completely analogous to the exceptional case above. The branching (2.17) contains two $\mathrm{SU}(6)$-singlets, but, by virtue of (2.16), one of the singlets can be freely acted on by the $\mathrm{U}(1)$. By acting with $G_{4} / \mathcal{G}_{1}=\mathrm{SO}(12) / \mathrm{SU}(6), \mathrm{Q}_{1}$ can be reduced to

$$
\mathbf{Q}_{1} \stackrel{\mathrm{SO}(12) / \mathrm{SU}(6)}{\longrightarrow}\left(I^{(1)}, \mathbf{0}_{\mathbf{1 5}}, \pm I^{(1)}, \mathbf{0}_{\mathbf{1 5}}\right),
$$

so that $I_{1}=1$, corresponding to the 1 -center quartic invariant $\mathcal{I}_{4}\left(\mathcal{Q}_{1}^{4}\right)$ only. Indeed, the general formula (2.2) yields

$$
I_{1}+\operatorname{dim}_{\mathbb{R}}(\mathrm{SO}(12))-\operatorname{dim}_{\mathbb{R}}(\mathrm{SU}(6))=1+66-35=32 .
$$

- Let us consider now the 2 -centered case $(p=2)$. Having solved the problem for $p=1$, we further decompose $\mathbf{Q}_{2}$ with respect to $\mathcal{G}_{1}=\mathrm{SU}(6)$ :

$$
\mathbf{Q}_{2}=\left(I^{(2)}, \mathbf{m}_{\mathbf{1 5}}, I^{(3)}, \mathbf{e}_{\mathbf{1 5}}\right) \text {, }
$$


and find the corresponding little group. The little group of the $\mathbf{1 5}$ of $\mathrm{SU}(6)$ is $U S p(6)$, under which such a representation branches as follows:

$$
15 \longrightarrow 1+14
$$

yielding the charge decompositions

$$
\mathbf{m}_{15} \longrightarrow\left(I^{(4)}, \mathbf{m}_{14}\right) ; \mathbf{e}_{15} \longrightarrow\left(I^{(5)}, \mathbf{e}_{14}\right) .
$$

Since $U S p(6)$ is maximally (and symmetrically) embedded in $S U$ (6), all singlets correspond to extra SO (12)-invariant polynomials, and $\mathbf{m}_{\mathbf{1 4}}$ can be set to zero through the action of $\mathcal{G}_{1} / U S p(6)=S U(6) / \mathrm{USp}(6)$, thus yielding the result:

$$
\mathbf{Q}_{2} \stackrel{S U(6) / \mathrm{USp}(6)}{\longrightarrow}\left(I^{(2)}, I^{(4)}, \mathbf{0}_{\mathbf{1 4}}, I^{(3)}, I^{(5)}, \mathbf{e}_{\mathbf{1 4}}\right) \text {. }
$$

- The 14 (rank-2 antisymmetric) of $\operatorname{USp}(6)$ has little group $[S U(2)]^{3}$, which does not commute with a $U(1)$ in USp(6). The $\mathbf{1 4}$ correspondingly branches as

$$
14 \longrightarrow(1,1,1)+(1,1,1)+(1,2,2)+(2,2,2),
$$

and thus

$$
\mathbf{e}_{14} \longrightarrow\left(I^{(6)}, I^{(7)}, \mathbf{e}_{(\mathbf{1}, 2,2)}, \mathbf{e}_{(\mathbf{2}, 2,2)}\right) \text {. }
$$

Therefore, by acting with $\operatorname{USp}(6) / \mathcal{G}_{2}=\mathrm{USp}(6) /[S U(2)]^{3}, \mathbf{Q}_{2}$ can then be put in the form

$$
\mathbf{Q}_{2} \stackrel{\mathrm{USp}(6) /[S U(2)]^{3}}{\longrightarrow}\left(I^{(2)}, I^{(4)}, \mathbf{0}_{\mathbf{1 4}}, I^{(3)}, I^{(5)}, I^{(6)}, I^{(7)}, \mathbf{0}_{(\mathbf{1 , 2 , 2})}, \mathbf{0}_{(\mathbf{2}, \mathbf{2}, \mathbf{2})}\right) .
$$

In conclusion, we found that the little group of a 2-centered black-hole solution is $\mathcal{G}_{2}=[S U(2)]^{3}$, and the corresponding 2-centered charge orbit reads (in complexified form)

$$
\mathcal{O}_{p=2}=\frac{G_{4}}{\mathcal{G}_{2}}=\frac{\mathrm{SO}(12)}{[S U(2)]^{3}} .
$$

The SO (12)-invariant polynomials for a 2-centered configuration are seven: $I_{2}=7$; indeed, the general formula (2.2) gives:

$$
I_{2}+\operatorname{dim}_{\mathbb{R}}(\operatorname{SO}(12))-\operatorname{dim}_{\mathbb{R}}\left([S U(2)]^{3}\right)=7+66-9=64=2 \cdot 32 .
$$

\section{$2.3 \quad J_{3}^{\mathbb{C}}(\mathcal{N}=2), M_{1,2}(\mathbb{O})(\mathcal{N}=5)$}

Let us now consider the model based on the Euclidean degree-3 Jordan algebra $J_{3}^{\mathbb{C}}$ on $\mathbb{C}$. Since, as mentioned earlier, we actually deal with groups on the complex field, this case pertains also to "pure" $\mathcal{N}=5$ supergravity, which is based on $M_{1,2}(\mathbb{O})$, the Jordan triple system (not upliftable to $d=5$ ) generated by $2 \times 1$ matrices over $\mathbb{O}[20,21]$.

In the complex field $G_{4}=S U(6)$, and $\mathbf{S y m p l}(S U(6))=\mathbf{2 0}$, the real self-dual rank-3 antisymmetric irreducible representation. 
- Let us first solve the problem for $p=1$. $\mathcal{G}_{1}$ is a real form of $\mathrm{SU}(3) \times \mathrm{SU}(3)$, the relevant (maximal symmetric) embedding is

$$
\mathrm{SU}(6) \supset \mathrm{SU}(3) \times \mathrm{SU}(3) \times \mathrm{U}(1)=\mathcal{G}_{1} \times \mathrm{U}(1)
$$

and the $\mathbf{2 0}$ accordingly branches as

$$
\mathbf{2 0} \rightarrow(\mathbf{1}, \mathbf{1})_{-3}+(\mathbf{3}, \overline{\mathbf{3}})_{-1}+(\overline{\mathbf{3}}, \mathbf{3})_{+1}+(\mathbf{1}, \mathbf{1})_{+3},
$$

corresponding to the charge decomposition

$$
\mathbf{Q}_{1} \rightarrow\left(p^{0}, \mathbf{p}_{(\mathbf{3}, \overline{\mathbf{3}})}, q_{0}, \mathbf{q}_{(\overline{\mathbf{3}}, \mathbf{3})}\right) .
$$

The analysis here is analogous to the cases treated above. The branching (2.31) contains two $[\mathrm{SU}(3) \times \mathrm{SU}(3)]$-singlets, but, by virtue of $(2.30)$, one of the singlets can be freely acted on by the $\mathrm{U}(1)$. By acting with $G_{4} / \mathcal{G}_{1}=S U(6) /[\mathrm{SU}(3) \times \mathrm{SU}(3)]$, $\mathbf{Q}_{1}$ can be reduced to

$$
\mathbf{Q}_{1} \stackrel{S U(6) /[\operatorname{SU}(3) \times \operatorname{SU}(3)]}{\longrightarrow}\left(I^{(1)}, \mathbf{0}_{(\mathbf{3}, \overline{\mathbf{3}})}, \pm I^{(1)}, \mathbf{0}_{(\overline{\mathbf{3}}, \mathbf{3})}\right),
$$

so that $I_{1}=1$, which corresponds to $\mathcal{I}_{4}\left(\mathcal{Q}_{1}^{4}\right)$ only. Indeed, formula (2.2) yields

$$
I_{1}+\operatorname{dim}_{\mathbb{R}}(S U(6))-\operatorname{dim}_{\mathbb{R}}(\mathrm{SU}(3) \times \mathrm{SU}(3))=1+35-16=20 .
$$

- Let us consider now the 2-centered case $(p=2)$. Having solved the problem for $p=1$, we further decompose $\mathbf{Q}_{2}$ with respect to $\mathcal{G}_{1}=\mathrm{SU}(3) \times \mathrm{SU}(3)$ :

$$
\mathbf{Q}_{2}=\left(I^{(2)}, \mathbf{m}_{(\mathbf{3}, \overline{\mathbf{3}})}, I^{(3)}, \mathbf{e}_{(\overline{\mathbf{3}}, \mathbf{3})}\right),
$$

and find the corresponding little group. The little group of the $(\mathbf{3}, \overline{\mathbf{3}})$ of $\mathrm{SU}(3) \times \mathrm{SU}(3)$ is the diagonal $\mathrm{SU}(3)$, which is maximal in $\mathrm{SU}(3) \times \mathrm{SU}(3)$ (see e.g. [32]), under which such a representation branches as follows:

$$
(\mathbf{3}, \overline{\mathbf{3}}) \rightarrow \mathbf{1}+\mathbf{8},
$$

yielding the charge decompositions

$$
\mathbf{m}_{(\mathbf{3}, \overline{\mathbf{3}})} \rightarrow\left(I^{(4)}, \mathbf{m}_{8}\right) ; \quad \mathbf{e}_{(\overline{\mathbf{3}}, \mathbf{3})} \rightarrow\left(I^{(5)}, \mathbf{e}_{8}\right) .
$$

The maximality of the embedding of the diagonal $\mathrm{SU}(3)$ in $\mathrm{SU}(3) \times \mathrm{SU}(3)$ implies all singlets to correspond to extra $S U(6)$-invariant polynomials, and $\mathbf{m}_{\mathbf{8}}$ can be set to zero through the action of $\mathcal{G}_{1} / \mathrm{SU}(3)=[\mathrm{SU}(3) \times \mathrm{SU}(3)] / \mathrm{SU}(3)$, thus yielding the result:

$$
\mathbf{Q}_{2} \stackrel{[\mathrm{SU}(3) \times \mathrm{SU}(3)] / \mathrm{SU}(3)}{\longrightarrow}\left(I^{(2)}, I^{(4)}, \mathbf{0}_{\mathbf{8}}, I^{(3)}, I^{(5)}, \mathbf{e}_{\mathbf{8}}\right) .
$$


- The $\mathbf{8}$ (adjoint) of $\mathrm{SU}(3)$ has little group $[\mathrm{U}(1)]^{2}$, which does not commute with any $\mathrm{U}(1)$ in $\mathrm{SU}(3)$. The 8 correspondingly branches as

$$
\mathbf{8} \rightarrow \mathbf{1}_{0,0}+\mathbf{1}_{0,0}+\mathbf{1}_{0,2}+\mathbf{1}_{0,-2}+\mathbf{1}_{3,1}+\mathbf{1}_{3,-1}+\mathbf{1}_{-3,1}+\mathbf{1}_{-3,-1},
$$

and thus

$$
\mathbf{e}_{\mathbf{8}} \longrightarrow\left(I^{(6)}, I^{(7)}, \mathrm{e}_{0,2}, \mathrm{e}_{0,-2}, \mathrm{e}_{3,1}, \mathrm{e}_{3,-1}, \mathrm{e}_{-3,1}, \mathrm{e}_{-3,-1}\right) .
$$

Therefore, by acting with $\mathrm{SU}(3) / \mathcal{G}_{2}=\mathrm{SU}(3) /[\mathrm{U}(1)]^{2}, \mathbf{Q}_{2}$ can then be put in the form

$$
\mathbf{Q}_{2} \stackrel{\mathrm{SU}(3) /[\mathrm{U}(1)]^{2}}{\longrightarrow}\left(I^{(2)}, I^{(4)}, \mathbf{0}_{\mathbf{8}}, I^{(3)}, I^{(5)}, I^{(6)}, I^{(7)}, \mathbf{0}_{6}\right),
$$

where $\mathbf{0}_{6}$ collectively denotes the six charges pertaining to the $[\mathrm{U}(1)]^{2}$-charged representations $\mathbf{1}_{0,2}, \mathbf{1}_{0,-2}, \mathbf{1}_{3,1}, \mathbf{1}_{3,-1}, \mathbf{1}_{-3,1}, \mathbf{1}_{-3,-1}$ in the right-hand side of (2.38).

In conclusion, we found that the little group of a 2-centered black-hole solution is $\mathcal{G}_{2}=[\mathrm{U}(1)]^{2}$, and the corresponding 2-centered charge orbit reads (in complexified form)

$$
\mathcal{O}_{p=2}=\frac{G_{4}}{\mathcal{G}_{2}}=\frac{S U(6)}{[\mathrm{U}(1)]^{2}}
$$

The $S U$ (6)-invariant polynomials for a 2-centered configuration are seven: $I_{2}=7$; indeed, the general formula (2.2) gives:

$$
I_{2}+\operatorname{dim}_{\mathbb{R}}(S U(6))-\operatorname{dim}_{\mathbb{R}}\left([\mathrm{U}(1)]^{2}\right)=7+35-2=40=2 \cdot 20 .
$$

\section{$2.4 J_{3}^{\mathbb{R}}(\mathcal{N}=2)$}

Finally, we consider the model based on the Euclidean degree-3 Jordan algebra $J_{3}^{\mathbb{R}}$ on $\mathbb{R}$.

In the complex field $G_{4}=U S p(6)$, and $\operatorname{Sympl}(U S p(6))=\mathbf{1 4}^{\prime}$, the real self-dual rank-3 antisymmetric irreducible representation of $U S p(6)$ (not to be confused with the rank-2 antisymmetric irreducible representation $\mathbf{1 4}$ considered in section 2.2).

- Let us first solve the problem for $p=1$. $\mathcal{G}_{1}$ is a real form of $\mathrm{SU}(3)$, the relevant (maximal symmetric) embedding is

$$
\mathrm{USp}(6) \supset \mathrm{SU}(3) \times \mathrm{U}(1)=\mathcal{G}_{1} \times \mathrm{U}(1)
$$

and the $14^{\prime}$ accordingly branches as

$$
14^{\prime} \rightarrow \mathbf{1}_{-3}+\mathbf{6}_{-1}+\overline{\mathbf{6}}_{+1}+\mathbf{1}_{+3},
$$

corresponding to the charge decomposition

$$
\mathbf{Q}_{1} \rightarrow\left(p^{0}, \mathbf{p}_{\mathbf{6}}, q_{0}, \mathbf{q}_{\mathbf{6}}\right) .
$$

Once again, the analysis here is analogous to the cases treated above. The branching (2.44) contains two $\mathrm{SU}(3)$-singlets, but, by virtue of (2.43), one of the singlets 
can be freely acted on by the $\mathrm{U}(1)$. By acting with $G_{4} / \mathcal{G}_{1}=U S p(6) / \mathrm{SU}(3), \mathrm{Q}_{1}$ can be reduced to

$$
\mathbf{Q}_{1} \stackrel{U S p(6) / S U(3)}{\longrightarrow}\left(I^{(1)}, \mathbf{0}_{\mathbf{6}}, \pm I^{(1)}, \mathbf{0}_{\mathbf{6}}\right),
$$

so that $I_{1}=1$, which corresponds to $\mathcal{I}_{4}\left(\mathcal{Q}_{1}^{4}\right)$ only. Indeed, formula (2.2) yields

$$
I_{1}+\operatorname{dim}_{\mathbb{R}}(U S p(6))-\operatorname{dim}_{\mathbb{R}}(\mathrm{SU}(3))=1+21-8=14 .
$$

- Let us consider now the 2-centered case $(p=2)$. Having solved the problem for $p=1$, we further decompose $\mathbf{Q}_{2}$ with respect to $\mathcal{G}_{1}=\mathrm{SU}(3)$ :

$$
\mathbf{Q}_{2}=\left(I^{(2)}, \mathbf{m}_{\mathbf{6}}, I^{(3)}, \mathbf{e}_{\mathbf{6}}\right),
$$

and find the corresponding little group. The little group of the $\mathbf{6}$ of SU(3) is $\mathrm{SO}(3)$, which is maximal in $\mathrm{SU}(3)$, under which such a representation branches as follows:

$$
6 \rightarrow 1+5
$$

yielding the charge decompositions

$$
\mathbf{m}_{\mathbf{6}} \rightarrow\left(I^{(4)}, \mathbf{m}_{\mathbf{5}}\right) ; \quad \mathbf{e}_{\mathbf{6}} \rightarrow\left(I^{(5)}, \mathbf{e}_{\mathbf{5}}\right) .
$$

The maximality of $\mathrm{SO}(3)$ in $\mathrm{SU}(3)$ implies all singlets to corresponds to extra USp (6)invariant polynomials, and $\mathbf{m}_{\mathbf{6}}$ can be set to zero through the action of $\mathcal{G}_{1} / \mathrm{SO}(3)=$ $\mathrm{SU}(3) / \mathrm{SO}(3)$, thus yielding the result:

$$
\mathbf{Q}_{2} \stackrel{\mathrm{SU}(3) / \mathrm{SO}(3)}{\longrightarrow}\left(I^{(2)}, I^{(4)}, \mathbf{0}_{\mathbf{5}}, I^{(3)}, I^{(5)}, \mathbf{e}_{\mathbf{5}}\right) .
$$

- Note, however, that the little group of the $\mathbf{5}$ (rank-2 symmetric traceless) irreducible representation of $\mathrm{SO}(3)$ is the identity, so that $\mathcal{G}_{2}=\mathbb{I}$. The $\mathbf{5}$ then trivially branches into five singlets, three of which can be rotated to zero through the action of $\mathrm{SO}(3) / \mathcal{G}_{2}=\mathrm{SO}(3)$ :

$$
\mathbf{Q}_{2} \stackrel{\mathrm{SO}(3)}{\longrightarrow}\left(I^{(2)}, I^{(4)}, \mathbf{0}_{\mathbf{5}}, I^{(3)}, I^{(5)}, I^{(6)}, I^{(7)}, \mathbf{0}_{3}\right),
$$

where $\mathbf{0}_{3}$ collectively denotes such three singlets set to zero.

In conclusion, we found that the little group of a 2-centered black-hole solution is the identity itself: $\mathcal{G}_{2}=\mathbb{I}$, and the corresponding 2 -centered charge orbit reads (in compact form)

$$
\mathcal{O}_{p=2}=\frac{G_{4}}{\mathcal{G}_{2}}=\operatorname{USp}(6) .
$$

The $U S p$ (6)-invariant polynomials for a 2-centered configuration are seven: $I_{2}=7$; indeed, the general formula (2.2) yields:

$$
I_{2}+\operatorname{dim}_{\mathbb{R}}(\mathrm{USp}(6))-\operatorname{dim}_{\mathbb{R}}(\mathbb{I})=7+21-0=28=2 \cdot 14 .
$$




\section{Invariant structures and the role of the horizontal symmetry $\mathrm{SL}_{h}(2, \mathbb{R})$}

We now propose a candidate for a complete basis of $G_{4}$-invariant polynomials for the $p=2$ case, highlighting the role of the horizontal symmetry group [18] in the classification of multi-center invariant structures.

Our treatment applies at least to the irreducible cubic geometries of symmetric scalar manifolds of $d=4$ supergravity theories [22] (which, with the exception of the rank-1 $t^{3}$ model ${ }^{5}$ are the ones considered in the counting analysis of section 2 ):

1. $\mathcal{N}=2$ magical Maxwell-Einstein supergravities $\left(J_{3}^{\mathbb{A}}, \mathbb{A}=\mathbb{O}, \mathbb{H}, \mathbb{C}, \mathbb{R}\right)$, with the case $J_{3}^{\mathbb{H}}$ encompassing also $\mathcal{N}=6$ "pure" supergravity [20, 21, 28-31];

2. $\mathcal{N}=5$ "pure" supergravity $\left(M_{1,2}(\mathbb{O})\right)$;

3. $\mathcal{N}=8$ "pure" supergravity $\left(J_{3}^{\mathbb{Q}_{s}}\right)$.

The simplest invariant structures of a simple Lie group $G$ (such as the $U$-duality group $G_{4}$ of an irreducible symmetric model) are the Killing-Cartan metric $g_{\alpha \beta}$, the structure constants $f_{\alpha \beta \gamma}$ and the symplectic metric $\mathbb{C}_{M N}$ (the Greek indices are in the adjoint representation of $G_{4}, \operatorname{Adj}\left(G_{4}\right)$, while the capital indices are in $\left.\operatorname{Sympl}\left(G_{4}\right)\right)$. It is well known that the entries of the generators in $\operatorname{Sympl}\left(G_{4}\right)$

$$
t_{\alpha \mid M N} \equiv t_{\alpha \mid M}^{P} \mathbb{C}_{P N}=t_{\alpha \mid(M N)}
$$

are invariant structures, symmetric in the symplectic indices (for the notation, see [33]).

In particular, one can construct the so-called $K$-tensor ${ }^{6}[34]$

$$
\mathbb{K}_{M N P Q} \equiv-\frac{1}{3 \tau} t_{(M N}^{\alpha} t_{\alpha \mid P Q)}=-\frac{1}{3 \tau}\left(t_{M N}^{\alpha} t_{\alpha \mid P Q}-\tau \mathbb{C}_{M(P} \mathbb{C}_{Q) N}\right)=\mathbb{K}_{(M N P Q)},
$$

where $\tau$ is a $G_{4}$-dependent constant defined as

$$
\tau \equiv \frac{2 d}{f(f+1)}
$$

with $d \equiv \operatorname{dim}_{\mathbb{R}} \mathbf{A d j}\left(G_{4}\right)$ and $f \equiv \operatorname{dim}_{\mathbb{R}}\left(\operatorname{Sympl}\left(G_{4}\right)\right)$. From its definition (3.2), the $K$-tensor is a completely symmetric rank- $4 G_{4}$-invariant tensor of $\operatorname{Sympl}\left(G_{4}\right)$.

In the presence of a single-centered black-hole background $(p=1)$, associated to a dyonic black-hole charge vector $\mathcal{Q}^{M}$ in $\operatorname{Sympl}\left(G_{4}\right)$, the unique independent $G_{4}$-invariant polynomial reads [34]

$$
\mathcal{I}_{4}\left(\mathcal{Q}^{4}\right) \equiv \mathbb{K}_{M N P Q} \mathcal{Q}^{M} \mathcal{Q}^{N} \mathcal{Q}^{P} \mathcal{Q}^{Q}=-\frac{1}{3 \tau} t_{M N}^{\alpha} t_{\alpha \mid P Q} \mathcal{Q}^{M} \mathcal{Q}^{N} \mathcal{Q}^{P} \mathcal{Q}^{Q}
$$

\footnotetext{
${ }^{5}$ As mentioned above, the irreducible rank-1 cubic case (the so-called $\mathcal{N}=2, d=4 t^{3}$ model, associated to the trivial degree-1 Jordan algebra $\mathbb{R}$ ) has been treated in [18].

${ }^{6}$ With respect to the treatment given in [34], we fix the overall normalization constant of the $K$-tensor to the value $\xi=-\frac{1}{3 \tau}=-\frac{f(f+1)}{6 d}$, as needed for consistency reasons.
} 
On the other hand, in the presence of a multi-centered black-hole solution $(p \geqslant 2)$, the horizontal symmetry $\mathrm{SL}_{h}(p, \mathbb{R})[18]$ plays a crucial role in organizing the various $G_{4^{-}}$ covariant and $G_{4}$-invariant structures.

In the following treatment we will consider the 2-centered case $(p=2)$, the index $a=1,2$ spanning the fundamental representation ( $\operatorname{spin} s=1 / 2) \mathbf{2}$ of the horizontal symmetry $\mathrm{SL}_{h}(2, \mathbb{R})$.

By using the symplectic representation (3.1) of the generators of $G_{4}$, one can introduce the tensor (homogeneous quadratic in charges)

$$
T_{\alpha \mid a b} \equiv t_{\alpha \mid M N} \mathcal{Q}_{a}^{M} \mathcal{Q}_{b}^{N}=T_{\alpha \mid(a b)}=\left(\begin{array}{cc}
T_{\alpha \mid 11} & T_{\alpha \mid 12} \\
T_{\alpha \mid 12} & T_{\alpha \mid 22}
\end{array}\right),
$$

lying in $\left(\mathbf{3}, \mathbf{A d j}\left(G_{4}\right)\right)$ of $\mathrm{SL}_{h}(2, \mathbb{R}) \times G_{4}$, where $\mathbf{3}$ is the rank-2 symmetric ( $\operatorname{spin} s=1$ ) representation of $\mathrm{SL}_{h}(2, \mathbb{R})$. In irreducible models, $T_{\alpha \mid a b}$ is the analogue of the so-called $\mathbb{T}$-tensor, introduced in [18] for reducible theories. Under the centers' exchange $1 \leftrightarrow 2$, $T_{\alpha \mid 11} \leftrightarrow T_{\alpha \mid 22}$, while $T_{\alpha \mid 12}$ is invariant.

Interestingly, one can prove that the quantity

$$
\mathbf{N} \equiv g^{\alpha \beta}\left(T_{\alpha \mid 11} T_{\beta \mid 22}-T_{\alpha \mid 12} T_{\beta \mid 12}\right)
$$

is not independent from lower order invariants. Indeed, at least in the aforementioned irreducible cases, it holds that

$$
t_{M[N}^{\alpha} t_{\alpha \mid P] Q}=\frac{\tau}{2}\left[\mathbb{C}_{M(P} \mathbb{C}_{Q) N}-\mathbb{C}_{M(N} \mathbb{C}_{Q) P}\right] .
$$

Thus, from (3.5) and (3.6), it follows that

$$
\mathbf{N}=2 t_{M[N}^{\alpha} t_{\alpha \mid P] Q} \mathcal{Q}_{1}^{M} \mathcal{Q}_{1}^{N} \mathcal{Q}_{2}^{P} \mathcal{Q}_{2}^{Q}=-\frac{1}{3}\left[\mathbb{C}_{M(P} \mathbb{C}_{Q) N}-\mathbb{C}_{M(N} \mathbb{C}_{Q) P}\right] \mathcal{Q}_{1}^{M} \mathcal{Q}_{1}^{N} \mathcal{Q}_{2}^{P} \mathcal{Q}_{2}^{Q}=\frac{1}{2} \mathcal{W}^{2}
$$

where

$$
\mathcal{W} \equiv\left\langle\mathcal{Q}_{1}, \mathcal{Q}_{2}\right\rangle \equiv \frac{1}{2} \mathbb{C}_{M N} \epsilon^{a b} \mathcal{Q}_{a}^{M} \mathcal{Q}_{b}^{N}
$$

is the symplectic product of the charge vectors $\mathcal{Q}_{1}$ and $\mathcal{Q}_{2}$, which is a singlet $(\mathbf{1}, \mathbf{1})$ of $\mathrm{SL}_{h}(2, \mathbb{R}) \times G_{4}$ (manifestly antisymmetric under $1 \leftrightarrow 2$ ).

An important difference between the reducible models (studied in [18]) and the irreducible treated in the present investigation is that, while the former generally have a non-vanishing horizontal invariant polynomial $\mathcal{X}$, the latter have it vanishing identically. Indeed, the analogue of $\mathcal{X}$ (defined by eq. (4.13) of [18]) for irreducible models can be defined as

$$
\mathcal{X}_{\text {irred }} \equiv \mathbf{N}-\frac{1}{2} \mathcal{W}^{2}=0,
$$

where result (3.8) was used in the last step.

By using the $K$-tensor (3.2), one can also define the tensor (homogeneous cubic in charges)

$$
\mathcal{Q}_{M \mid a b c} \equiv \mathbb{K}_{M N P Q} \mathcal{Q}_{a}^{N} \mathcal{Q}_{b}^{P} \mathcal{Q}_{c}^{Q}=\mathcal{Q}_{M \mid(a b c)},
$$


lying in $\left(\mathbf{4}, \operatorname{Sympl}\left(G_{4}\right)\right)$ of $\mathrm{SL}_{h}(2, \mathbb{R}) \times G_{4}$, where $\mathbf{4}$ is the rank-3 symmetric representation ( $\operatorname{spin} s=3 / 2$ ) of $\mathrm{SL}_{h}(2, \mathbb{R})$. Under $1 \leftrightarrow 2$, it holds that $\mathcal{Q}_{M \mid 111} \leftrightarrow \mathcal{Q}_{M \mid 222}$ and $\mathcal{Q}_{M \mid 112} \leftrightarrow \mathcal{Q}_{M \mid 122}$.

By further contracting with a 2-centered charge vector, one can introduce the tensor (homogeneous quartic in charges)

$$
\mathbf{I}_{a b c d} \equiv \mathbb{K}_{M N P Q} \mathcal{Q}_{a}^{M} \mathcal{Q}_{b}^{N} \mathcal{Q}_{c}^{P} \mathcal{Q}_{d}^{Q}=\mathbf{I}_{(a b c d)}
$$

lying in $(\mathbf{5}, \mathbf{1})$ of $\mathrm{SL}_{h}(2, \mathbb{R}) \times G_{4}$, where $\mathbf{5}$ is the rank-4 symmetric representation (spin $s=2)$ of $\mathrm{SL}_{h}(2, \mathbb{R})$. Under $1 \leftrightarrow 2, \mathbf{I}_{1111} \leftrightarrow \mathbf{I}_{2222}, \mathbf{I}_{1112} \leftrightarrow \mathbf{I}_{1222}$, while $\mathbf{I}_{1122}$ is invariant.

Trivially, $\widetilde{\mathcal{Q}}_{a b c} \equiv \mathcal{Q}_{M \mid a b c}$ and $\mathbf{I}_{(a b c d)}$ are related by ${ }^{7}$

$$
\begin{aligned}
\mathbf{I}_{a b c d} & =\mathcal{Q}_{M \mid a b c} \mathcal{Q}_{d}^{M}=\mathbb{C}^{M N} \mathcal{Q}_{M \mid a b c} \mathcal{Q}_{N \mid d}=\left\langle\widetilde{\mathcal{Q}}_{a b c}, \mathcal{Q}_{d}\right\rangle ; \\
\mathcal{Q}_{M \mid a b c} & =\frac{1}{4} \frac{\partial \mathbf{I}_{a b c d}}{\partial \mathcal{Q}_{d}^{M}} .
\end{aligned}
$$

Note that only the completely symmetric part $\mathcal{Q}_{M \mid(a b c} \mathcal{Q}_{d)}^{M}$ survives the contraction in (3.13), because $\mathcal{Q}_{M \mid a b c} \mathcal{Q}_{d}^{M} \epsilon^{c d}=0$ from the symmetry of the $K$-tensor (3.2) and the definition (3.11) of $\mathcal{Q}_{M \mid a b c}$ itself.

In order to generate $G_{4}$-invariant polynomials, one can:

1. multiply and contract on $\mathbf{A d j}\left(G_{4}\right)$ the three components of the quadratic tensor $T_{\alpha \mid a b}$ defined by (3.5), or

2. contract all four components of $\mathcal{Q}_{M \mid a b c}$ defined by (3.11) with three 2-center charge vectors, in all possible ways, or

3. contract all five components of $\mathbf{I}_{a b c d}$ defined by (3.12) with four 2-center charge vectors, in all possible ways.

By virtue of the various relations considered above, these three approaches give equivalent results, which we now specify for the sake of clarity:

$$
\begin{aligned}
\mathbf{I}_{+2}\left(\mathcal{Q}_{1}^{4}\right) & \equiv \mathcal{I}_{4}\left(\mathcal{Q}_{1}^{4}\right) \equiv \mathbf{I}_{1111} \\
& =\left\langle\widetilde{\mathcal{Q}}_{111}, \mathcal{Q}_{1}\right\rangle=\mathbb{K}_{M N P Q} \mathcal{Q}_{1}^{M} \mathcal{Q}_{1}^{N} \mathcal{Q}_{1}^{P} \mathcal{Q}_{1}^{Q}=-\frac{1}{3 \tau} T_{11}^{\alpha} T_{\alpha \mid 11} \\
\mathbf{I}_{+1}\left(\mathcal{Q}_{1}^{3} \mathcal{Q}_{2}\right) & \equiv \mathbf{I}_{1112} \\
& =\left\langle\widetilde{\mathcal{Q}}_{111}, \mathcal{Q}_{2}\right\rangle=\left\langle\widetilde{\mathcal{Q}}_{112}, \mathcal{Q}_{1}\right\rangle=\mathbb{K}_{M N P Q} \mathcal{Q}_{1}^{M} \mathcal{Q}_{1}^{N} \mathcal{Q}_{1}^{P} \mathcal{Q}_{2}^{Q}=-\frac{1}{3 \tau} T_{11}^{\alpha} T_{12 \mid \alpha}
\end{aligned}
$$

\footnotetext{
${ }^{7}$ We remark that relation (3.14) characterizes $\widetilde{\mathcal{Q}}_{a b c}$ as the 2-center generalisation of the so-called Freudenthal dual of the dyonic charge vector $\mathcal{Q}^{M}$, introduced (with a different normalisation) in [36]. Thus, $\widetilde{\mathcal{Q}}_{a b c}$ can be regarded as the (polynomial) 2-center Freudenthal dual of the dyonic charge vector $\mathcal{Q}_{d}$.

Furthermore, eqs. (3.9), (3.13) and (3.24) yield that, under the formal interchange $\mathcal{Q}_{a}^{M} \leftrightarrow \mathbb{C}^{M N} \mathcal{Q}_{N \mid a b c}$, $\mathbf{I}_{a b c d}$ is invariant and $\mathcal{W} \leftrightarrow \mathbf{I}_{6}$.
} 


$$
\begin{aligned}
\mathbf{I}_{0}\left(\mathcal{Q}_{1}^{2} \mathcal{Q}_{2}^{2}\right) & \equiv \mathbf{I}_{1122} \\
& =\left\langle\widetilde{\mathcal{Q}}_{112}, \mathcal{Q}_{2}\right\rangle=\left\langle\widetilde{\mathcal{Q}}_{122}, \mathcal{Q}_{1}\right\rangle=\mathbb{K}_{M N P Q} \mathcal{Q}_{1}^{M} \mathcal{Q}_{1}^{N} \mathcal{Q}_{2}^{P} \mathcal{Q}_{2}^{Q} \\
& =-\frac{1}{9 \tau}\left(T_{11}^{\alpha} T_{22 \mid \alpha}+2 T_{12}^{\alpha} T_{12 \mid \alpha}\right)=-\frac{1}{3 \tau}\left(T_{11}^{\alpha} T_{22 \mid \alpha}+\tau \mathcal{W}^{2}\right) \\
\mathbf{I}_{-1}\left(\mathcal{Q}_{1} \mathcal{Q}_{2}^{3}\right) & \equiv \mathbf{I}_{1222} \\
& =\left\langle\widetilde{\mathcal{Q}}_{122}, \mathcal{Q}_{2}\right\rangle=\left\langle\widetilde{\mathcal{Q}}_{222}, \mathcal{Q}_{1}\right\rangle=\mathbb{K}_{M N P Q} \mathcal{Q}_{1}^{M} \mathcal{Q}_{2}^{N} \mathcal{Q}_{2}^{P} \mathcal{Q}_{2}^{Q}=-\frac{1}{3 \tau} T_{22}^{\alpha} T_{12 \mid \alpha} \\
\mathbf{I}_{-2}\left(\mathcal{Q}_{2}^{4}\right) & \equiv \mathcal{I}_{4}\left(\mathcal{Q}_{2}^{4}\right) \equiv \mathbf{I}_{2222} \\
& =\left\langle\widetilde{\mathcal{Q}}_{222}, \mathcal{Q}_{2}\right\rangle=\mathbb{K}_{M N P Q} \mathcal{Q}_{2}^{M} \mathcal{Q}_{2}^{N} \mathcal{Q}_{2}^{P} \mathcal{Q}_{2}^{Q}=-\frac{1}{3 \tau} T_{22}^{\alpha} T_{22 \mid \alpha}
\end{aligned}
$$

The subscripts in the $G_{4}$-invariant polynomials $\mathbf{I}_{+2}, \mathbf{I}_{+1}, \mathbf{I}_{0}, \mathbf{I}_{-1}$ and $\mathbf{I}_{-2}$ defined by (3.15)(3.19) denote the polarization with respect to the horizontal symmetry $\mathrm{SL}_{h}(2, \mathbb{R})$, inherited from the components of $\mathbf{I}_{a b c d}(3.12)$; indeed, the five $G_{4}$-invariant polynomials (3.15)-(3.19) sit in the rank-4 symmetric representation ( $\operatorname{spin} s=2) \mathbf{5}$ of $\mathrm{SL}_{h}(2, \mathbb{R})$ itself [18].

In order to proceed further, it is worth mentioning the decomposition [34]

$$
t_{\alpha \mid M}{ }^{N} t_{\beta \mid N Q}=-t_{\alpha \mid M P} t_{\beta \mid N Q} \mathbb{C}^{P N}=\frac{1}{2 n} g_{\alpha \beta} \mathbb{C}_{M Q}+\frac{1}{2} f_{\alpha \beta}{ }^{\gamma} t_{\gamma \mid M Q}+S_{(\alpha \beta)[M Q]},
$$

where

$$
S_{\alpha \beta \mid M N}=S_{(\alpha \beta) \mid[M N]}
$$

denotes an invariant primitive tensor of $G_{4}$. From (3.20), the following identity for the $K$-tensor can be derived [34] (recall footnote 6):

$$
\begin{aligned}
\mathbb{K}_{M N P Q} \mathbb{K}_{R S T U} \mathbb{C}^{Q R}= & -\frac{(f+1)}{6 d} \mathbb{K}_{(M N \mid(S T} \mathbb{C}_{U) \mid P)}+\frac{(f+1)}{18 d} \mathbb{C}_{(M \mid(S \mid} \mathbb{C}_{|N||T|} \mathbb{C}_{\mid P) \mid U)} \\
& +\frac{f^{2}(f+1)^{2}}{72 d^{2}} f_{\alpha \beta \gamma} t_{(M N}^{\alpha} t_{P)(S} t_{T U)}^{\gamma} \\
& -\frac{f^{2}(f+1)^{2}}{36 d^{2}} t_{(M N}^{\alpha} S_{\alpha \beta \mid P)(S} t_{T U)}^{\beta},
\end{aligned}
$$

where

$$
S_{\alpha \beta \mid 12} \equiv S_{\alpha \beta \mid M N} \mathcal{Q}_{1}^{M} \mathcal{Q}_{2}^{N}=S_{\alpha \beta \mid M N} \mathcal{Q}_{1}^{[M} \mathcal{Q}_{2}^{N]}=-S_{\alpha \beta \mid 21} .
$$

A $G_{4}$-invariant polynomial homogeneous sextic in charges can then be defined as follows:

$$
\begin{aligned}
\mathbf{I}_{6}\left(\mathcal{Q}_{1}^{3} \mathcal{Q}_{2}^{3}\right) \equiv & \frac{1}{8}\left\langle\widetilde{\mathcal{Q}}_{a b c}, \widetilde{\mathcal{Q}}_{d e f}\right\rangle \epsilon^{a d} \epsilon^{b e} \epsilon^{c f}=\frac{1}{8} \mathbb{C}^{M N} \mathcal{Q}_{M \mid a b c} \mathcal{Q}_{N \mid d e f} \epsilon^{a d} \epsilon^{b e} \epsilon^{c f} \\
= & \frac{1}{4}\left\langle\widetilde{\mathcal{Q}}_{111}, \widetilde{\mathcal{Q}}_{222}\right\rangle+\frac{3}{4}\left\langle\widetilde{\mathcal{Q}}_{122}, \widetilde{\mathcal{Q}}_{112}\right\rangle \\
= & \frac{1}{4} \mathbb{K}_{M N P Q} \mathbb{K}_{R S T U} \mathbb{C}^{Q R}\left(\mathcal{Q}_{1}^{M} \mathcal{Q}_{1}^{N} \mathcal{Q}_{1}^{P} \mathcal{Q}_{2}^{S} \mathcal{Q}_{2}^{T} \mathcal{Q}_{2}^{U}+3 \mathcal{Q}_{1}^{M} \mathcal{Q}_{2}^{N} \mathcal{Q}_{2}^{P} \mathcal{Q}_{1}^{S} \mathcal{Q}_{1}^{T} \mathcal{Q}_{2}^{U}\right) \\
= & \frac{(f+1)}{36 d} \mathcal{W}^{3}+\frac{f^{2}(f+1)^{2}}{144 d^{2}} f_{\alpha \beta \gamma} T_{11}^{\alpha} T_{12}^{\beta} T_{22}^{\gamma} \\
& +\frac{f^{2}(f+1)^{2}}{108 d^{2}}\left(T_{12}^{\alpha} T_{12}^{\beta}-T_{11}^{\alpha} T_{22}^{\beta}\right) S_{\alpha \beta \mid 12} .
\end{aligned}
$$


Note that $\mathbf{I}_{6}$ is manifestly antisymmetric under $1 \leftrightarrow 2$. The first line of (3.24) is manifestly $\left[\mathrm{SL}_{h}(2, \mathbb{R}) \times G_{4}\right]$-invariant, the second and third lines provide explicit expressions, and in the fourth line the "master" identity (3.22) was exploited.

If the symplectic product $\mathcal{W} \neq 0$ (defined in (3.9)), the two charge vectors $\mathcal{Q}_{1}^{M}$ and $\mathcal{Q}_{2}^{M}$ are mutually non-local. The concept of mutual non-locality is very important in the treatment of marginal stability in multi-center black holes (see e.g. [1-3, 10-12, 24, 25, 35]).

The above treatment suggests that a candidate for a complete basis of $G_{4}$-invariant polynomials in the irreducible cases under consideration is given by the seven polynomials:

$$
\left(\mathcal{W}, \mathbf{I}_{+2}, \mathbf{I}_{+1}, \mathbf{I}_{0}, \mathbf{I}_{-1}, \mathbf{I}_{-2}, \mathbf{I}_{6}\right)
$$

respectively defined by (3.9), (3.15)-(3.19) and (3.24). The corresponding candidate for a complete basis of $\left[\mathrm{SL}_{h}(2, \mathbb{R}) \times G_{4}\right]$-invariant polynomials in the irreducible cases under consideration is then given by the four polynomials

$$
\left(\mathcal{W}, \mathbf{I}_{6}, \operatorname{Tr}\left(\mathbf{I}^{2}\right), \operatorname{Tr}\left(\mathbf{I}^{3}\right)\right)
$$

where [18]

$$
\begin{aligned}
& \operatorname{Tr}\left(\mathbf{I}^{2}\right)=\mathbf{I}_{+2} \mathbf{I}_{-2}+3 \mathbf{I}_{0}^{2}-4 \mathbf{I}_{+1} \mathbf{I}_{-1} ; \\
& \operatorname{Tr}\left(\mathbf{I}^{3}\right)=\mathbf{I}_{0}^{3}+\mathbf{I}_{+2} \mathbf{I}_{-1}^{2}+\mathbf{I}_{-2} \mathbf{I}_{+1}^{2}-\mathbf{I}_{+2} \mathbf{I}_{-2} \mathbf{I}_{0}-2 \mathbf{I}_{+1} \mathbf{I}_{0} \mathbf{I}_{-1} .
\end{aligned}
$$

Indeed, the spin $s=2$ representation 5 of $\operatorname{SL}_{h}(2, \mathbb{R})$, whose components are the $G_{4^{-}}$ invariant polynomials $\mathbf{I}_{+2}, \mathbf{I}_{+1}, \mathbf{I}_{0}, \mathbf{I}_{-1}$ and $\mathbf{I}_{-2}$ (defined by (3.15)-(3.19)), can be rearranged as a $3 \times 3$ symmetric traceless matrix $\mathbf{I}$ [18]. (3.27) and (3.28) (respectively homogeneous of order eight and twelve in charges) are the only independent $\mathrm{SL}_{h}(2, \mathbb{R})$-singlets which can be built out of such a $3 \times 3$ symmetric matrix I, due to its tracelessness [18]. Note that $\operatorname{Tr}\left(\mathbf{I}^{2}\right)$ and $\operatorname{Tr}\left(\mathbf{I}^{3}\right)$ are both invariant under $1 \leftrightarrow 2$.

It is worth pointing out that the analysis of sections 2 and 3 can be easily generalised to $p \geqslant 3$ centers. The two-centered representation of $\operatorname{spin} s=J / 2$ of $\mathrm{SL}_{h}(2, \mathbb{R})$ is then replaced by the completely symmetric rank- $J$ tensor representation $\mathcal{R}_{J}$ of $\operatorname{SL}_{h}(p, \mathbb{R})(J=1,2,3,4$ are the values relevant for the above analysis). On the other hand, $\mathcal{W}$ and $\mathbf{I}_{6}$ generally sit in the $\left(\widetilde{\mathcal{R}}_{2}, \mathbf{1}\right)$ representation of $\mathrm{SL}_{h}(p, \mathbb{R}) \times G_{4}$, where $\widetilde{\mathcal{R}}_{2}$ is the rank-2 antisymmetric representation of $\mathrm{SL}_{h}(p, \mathbb{R})$ (which, in the case $p=2$, becomes a singlet). However, due to the tree structure of the split flow in multi-center supergravity solutions [1-3, 10-12], to consider only the case $p=2$ does not imply any loss in generality (as far as marginal stability issues are concerned).

\section{Two-centered orbits with non-compact stabiliser: the $\mathcal{N}=8$ BPS and octonionic $\mathcal{N}=2$ non-BPS cases}

For $\mathcal{N}=2$ BPS two-centered extremal black holes, the stabiliser of the supporting charge orbit is always compact, so the orbit is unique (see table 1 for magical models). This is no longer the case when the stabiliser is non-compact, as it holds for $\mathcal{N}=2$ two-centered 
solutions with two non-BPS centers characterised by $\mathcal{I}_{4}\left(\mathcal{Q}_{1}^{4}\right)>0$ and $\mathcal{I}_{4}\left(\mathcal{Q}_{2}^{4}\right)>0$, and for $\mathcal{N} \geqslant 3$ two-centered solutions with two $\frac{1}{\mathcal{N}}$-BPS centers. These are interesting cases, in which a split attractor flow through a wall of marginal stability has been shown to occur $[37,38]$.

We will consider here the $\frac{1}{8}$ - BPS two-centered orbits in the maximal $\mathcal{N}=8$ theory (based on $J_{3}^{\mathbb{Q}_{s}}$ ) and the non-BPS two-centered orbits (of the aforementioned type) in the exceptional $\mathcal{N}=2$ magic model, based on $J_{3}^{\mathbb{Q}}$. These two cases can be obtained by repeating the analysis of section 2.1 and choosing suitable non-compact real forms of $G_{4}$ and $\mathcal{G}_{2}$.

The 1-centered charge orbits respectively read [23, 39]:

$$
\begin{aligned}
& \mathcal{N}=8, \frac{1}{8} \text {-BPS : } \mathcal{O}_{p=1}=\frac{E_{7(7)}}{E_{6(2)}} ; \\
& \mathcal{N}=2, J_{3}^{\mathbb{Q}} \operatorname{nBPS} \mathcal{I}_{4}>0: \mathcal{O}_{p=1}=\frac{E_{7(-25)}}{E_{6(-14)}} .
\end{aligned}
$$

In the maximal case, the chain of relevant group branchings reads

$$
\mathcal{N}=8, \frac{1}{8} \text {-BPS }: E_{7(7)} \longrightarrow E_{6(2)} \longrightarrow F_{4(4)} \longrightarrow \mathrm{SO}(5,4) \longrightarrow\left\{\begin{array}{l}
\mathrm{SO}(4,4) \\
\text { or } \\
\mathrm{SO}(5,3)
\end{array},\right.
$$

such that two $\frac{1}{8}$-BPS, $\mathcal{N}=8$, 2-centered charge orbits exist:

$$
\begin{aligned}
\mathcal{O}_{\mathcal{N}=8, \frac{1}{8}-\mathrm{BPS}, p=2, \mathbf{I}} & =\frac{E_{7(7)}}{\mathrm{SO}(4,4)} \\
\mathcal{O}_{\mathcal{N}=8, \frac{1}{8}-\mathrm{BPS}, p=2, \mathbf{I I}} & =\frac{E_{7(7)}}{\mathrm{SO}(5,3)} .
\end{aligned}
$$

In the $\mathcal{N}=2$ exceptional case, the chain of relevant group branchings reads

$$
\mathcal{N}=2, J_{3}^{\mathbb{Q}} \mathrm{nBPS}: E_{7(-25)} \longrightarrow E_{6(-14)} \longrightarrow F_{4(-20)} \longrightarrow\left\{\begin{array}{l}
\mathrm{SO}(9) \longrightarrow \mathrm{SO}(8) \\
\text { or } \\
\mathrm{SO}(8) \\
\mathrm{SO}(8,1) \longrightarrow \text { or } \\
\mathrm{SO}(7,1)
\end{array}\right.
$$

such that two non-BPS, $\mathcal{N}=2$, 2-centered charge orbits exist:

$$
\begin{gathered}
\mathcal{O}_{\mathcal{N}=2, J_{3}^{\oplus}, \mathrm{nBPS}, p=2, \mathbf{I}}=\frac{E_{7(-25)}}{\mathrm{SO}(8)} \\
\mathcal{O}_{\mathcal{N}=2, J_{3}, \mathrm{nBPS}, p=2, \mathbf{I I}}=\frac{E_{7(-25)}}{\mathrm{SO}(7,1)} .
\end{gathered}
$$

As it holds for the stabilizer of $\mathcal{O}_{\mathcal{N}=2, J_{3}^{\circledR}, \text { BPS, } p=2}$ (see table 1), the Lie algebra $\mathfrak{s o}$ (8) of the stabilizer of $\mathcal{O}_{\mathcal{N}=2, J_{3}^{\oplus}, \text { nBPS, } p=2, \mathbf{I}}(4.7)$ is nothing but the Lie algebra tri $(\mathbb{O})$ of the automorphism group $A u t(\mathbf{t}(\mathbb{O}))$ of the normed triality over the octonionic division algebra 
(1) (see e.g. eq. (21) of [27]). It is here worth observing that the Lie algebra $\mathfrak{s o}(4,4)$ of the stabilizer of $\mathcal{O}_{\mathcal{N}=8, \frac{1}{8}-\mathrm{BPS}, p=2, \mathbf{I}}$ (4.4) enjoys an analogous interpretation as the Lie algebra $\operatorname{tri}\left(\mathbb{O}_{s}\right)$ of the automorphism group Aut $\left(\mathbf{t}\left(\mathbb{O}_{s}\right)\right)$ of the normed triality over the split form $\mathbb{O}_{s}$ of the octonions. On the other hand, a similar interpretation seems not to hold for the stabilizer of $\mathcal{O}_{\mathcal{N}=8, \frac{1}{8} \text {-BPS, } p=2, \mathbf{I I}}(4.5)$ as well as for the stabilizer of $\mathcal{O}_{\mathcal{N}=2, J_{3}^{\oplus}, \text { nBPS, } p=2, \mathbf{I I}}$ (4.8).

We expect the $\mathcal{N}=8$ orbits (4.4) and (4.5), as well as the $\mathcal{N}=2$ orbits (4.7) and (4.8), to be defined by different constraints on the four $\mathrm{SL}_{h}(2, \mathbb{R}) \times G_{4}$ invariant polynomials given by eq. (3.26); we leave this interesting issue for further future investigation.

Here, we confine ourselves to present parallel results on pseudo-orthogonal groups, which may shed some light on the whole framework. Let us consider two vectors $\mathbf{x}$ and $\mathbf{y}$ in a pseudo-Euclidean $(p+q)$-dimensional space $E_{p, q}$ with signature $(p, q)$ and $p>1$, $q>1$. The norm of a vector is defined as, say

$$
\mathbf{x}^{2} \equiv x_{1}^{2}+\ldots+x_{p}^{2}-x_{p+1}^{2}-\ldots-x_{p+q}^{2}
$$

and the scalar product as

$$
\mathbf{x} \cdot \mathbf{y} \equiv x_{1} y_{1}+\ldots+x_{p} y_{p}-x_{p+1} y_{p+1}-\ldots-x_{p+q} y_{p+q} .
$$

The one-vector orbits (for non-lightlike vectors) are well

$$
\begin{aligned}
\mathcal{O}_{p=1, \text { timelike }} & =\frac{\mathrm{SO}(p, q)}{\mathrm{SO}(p-1, q)} \text { if } \mathbf{x}^{2}>0 \\
\mathcal{O}_{p=1, \text { spacelike }} & =\frac{\mathrm{SO}(p, q)}{\mathrm{SO}(p, q-1)} \text { if } \mathbf{x}^{2}<0 .
\end{aligned}
$$

It is intuitively clear that the two-vector orbits do depend on the nature of the vectors themselves. Let us start and consider two timelike vectors $\left(\mathbf{x}^{2}>0\right.$ and $\left.\mathbf{y}^{2}>0\right)$, whose

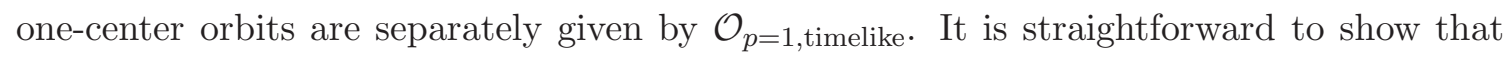
the two-center orbits supporting this configuration are

$$
\begin{gathered}
\frac{\mathrm{SO}(p, q)}{\mathrm{SO}(p-2, q)} \text { if } \mathbf{x}^{2} \mathbf{y}^{2}>(\mathbf{x} \cdot \mathbf{y})^{2} ; \\
\frac{\mathrm{SO}(p, q)}{\mathrm{SO}(p-1, q-1)} \text { if } \mathbf{x}^{2} \mathbf{y}^{2}<(\mathbf{x} \cdot \mathbf{y})^{2} .
\end{gathered}
$$

If both vectors are spacelike $\left(\mathbf{x}^{2}<0\right.$ and $\left.\mathbf{y}^{2}<0\right)$, the two-center orbits read

$$
\begin{gathered}
\frac{\mathrm{SO}(p, q)}{\mathrm{SO}(p, q-2)} \text { if } \mathbf{x}^{2} \mathbf{y}^{2}>(\mathbf{x} \cdot \mathbf{y})^{2} \\
\frac{\mathrm{SO}(p, q)}{\mathrm{SO}(p-1, q-1)} \text { if } \mathbf{x}^{2} \mathbf{y}^{2}<(\mathbf{x} \cdot \mathbf{y})^{2} .
\end{gathered}
$$

Finally, if one vector is timelike and the other one is spacelike (say, $\mathbf{x}^{2}>0$ and $\mathbf{y}^{2}<0$ ), the two-center orbit is unique:

$$
\frac{\mathrm{SO}(p, q)}{\mathrm{SO}(p-1, q-1)}
$$


because in this case $\mathbf{x}^{2} \mathbf{y}^{2}<(\mathbf{x} \cdot \mathbf{y})^{2}$ always holds.

By introducing the $\mathrm{SL}_{h}(2, R) \times \mathrm{SO}(p, q)$ invariant polynomial (see [40-43] and the last ref. of $[4-7])$

$$
\mathbf{I}_{4}(\mathbf{x}, \mathbf{y}) \equiv \mathbf{x}^{2} \mathbf{y}^{2}-(\mathbf{x} \cdot \mathbf{y})^{2},
$$

all orbits (4.13)-(4.17) can actually be recognised to correspond to only three orbits (namely $(4.13),(4.15)$, and $(4.14)=(4.16)=(4.17))$, respectively defined by the $\left[\mathrm{SL}_{h}(2, R) \times \mathrm{SO}(p, q)\right]$-invariant constraints: $\mathbf{I}_{4}>0\left(\right.$ with $\mathbf{x}^{2}>0$ and $\left.\mathbf{y}^{2}>0\right) ; \mathbf{I}_{4}>0$ (with $\mathbf{x}^{2}<0$ and $\mathbf{y}^{2}<0$ ); $\mathbf{I}_{4}<0$. Note that in the compact case (Euclidean signature: $q=0) \mathbf{I}_{4}>0$ due to the Cauchy-Schwarz triangular inequality, and the two-vector orbit is unique: $\frac{\mathrm{SO}(p)}{\mathrm{SO}(p-2)}$. This is in analogy with the results (obtained in the complex field) discussed in section 2 .

\section{Acknowledgments}

S. F. and A. M. would like to thank Raymond Stora, Emanuele Orazi and Armen Yeranyan for useful discussions.

The work of S. F. is supported by the ERC Advanced Grant no. 226455, "Supersymmetry, Quantum Gravity and Gauge Fields" (SUPERFIELDS), and in part by DOE Grant DE-FG03-91ER40662.

Open Access. This article is distributed under the terms of the Creative Commons Attribution Noncommercial License which permits any noncommercial use, distribution, and reproduction in any medium, provided the original author(s) and source are credited.

\section{References}

[1] F. Denef and G.W. Moore, Split states, entropy enigmas, holes and halos, hep-th/0702146 [SPIRES].

[2] F. Denef, D. Gaiotto, A. Strominger, D. Van den Bleeken and X. Yin, Black hole deconstruction, hep-th/0703252 [SPIRES].

[3] F. Denef and G.W. Moore, How many black holes fit on the head of a pin?, Int. J. Mod. Phys. D 17 (2008) 679 [arXiv:0705.2564] [SPIRES].

[4] S. Ferrara, R. Kallosh and A. Strominger, $N=2$ extremal black holes, Phys. Rev. D 52 (1995) 5412 [hep-th/9508072] [SPIRES].

[5] A. Strominger, Macroscopic entropy of $n=2$ extremal black holes, Phys. Lett. B 383 (1996) 39 [hep-th/9602111] [SPIRES].

[6] S. Ferrara and R. Kallosh, Supersymmetry and attractors, Phys. Rev. D 54 (1996) 1514 [hep-th/9602136] [SPIRES].

[7] S. Ferrara and R. Kallosh, Universality of supersymmetric attractors, Phys. Rev. D 54 (1996) 1525 [hep-th/9603090] [SPIRES].

[8] S. Ferrara, G.W. Gibbons and R. Kallosh, Black holes and critical points in moduli space, Nucl. Phys. B 500 (1997) 75 [hep-th/9702103] [SPIRES]. 
[9] L. Andrianopoli, R. D'Auria, S. Ferrara and M. Trigiante, Extremal black holes in supergravity, Lect. Notes Phys. 737 (2008) 661 [hep-th/0611345] [SPIRES].

[10] F. Denef, Supergravity flows and D-brane stability, JHEP 08 (2000) 050 [hep-th/0005049] [SPIRES].

[11] F. Denef, B.R. Greene and M. Raugas, Split attractor flows and the spectrum of BPS D-branes on the quintic, JHEP 05 (2001) 012 [hep-th/0101135] [SPIRES].

[12] B. Bates and F. Denef, Exact solutions for supersymmetric stationary black hole composites, hep-th/0304094 [SPIRES].

[13] E. Cremmer and B. Julia, The $N=8$ supergravity theory. 1. The lagrangian, Phys. Lett. B 80 (1978) 48 [SPIRES].

[14] E. Cremmer and B. Julia, The SO(8) supergravity, Nucl. Phys. B 159 (1979) 141 [SPIRES].

[15] C.M. Hull and P.K. Townsend, Unity of superstring dualities, Nucl. Phys. B 438 (1995) 109 [hep-th/9410167] [SPIRES].

[16] J.R. David, On walls of marginal stability in $N=2$ string theories, JHEP 08 (2009) 054 [arXiv:0905.4115] [SPIRES].

[17] S. Ferrara, A. Marrani and E. Orazi, Split attractor flow in $N=2$ minimally coupled supergravity, Nucl. Phys. B 846 (2011) 512 [arXiv:1010.2280] [SPIRES].

[18] S. Ferrara, A. Marrani, E. Orazi, R. Stora and A. Yeranyan, Two-center black holes duality-invariants for STU model and its lower-rank descendants, arXiv:1011.5864 [SPIRES].

[19] J.F. Luciani, Coupling of $\mathrm{O}(2)$ supergravity with several vector multiplets, Nucl. Phys. B 132 (1978) 325 [SPIRES].

[20] M. Günaydin, G. Sierra and P.K. Townsend, Exceptional supergravity theories and the MAGIC square, Phys. Lett. B 133 (1983) 72 [SPIRES].

[21] M. Günaydin, G. Sierra and P.K. Townsend, The geometry of $N=2$ Maxwell-Einstein supergravity and Jordan algebras, Nucl. Phys. B 242 (1984) 244 [SPIRES].

[22] B. de Wit, F. Vanderseypen and A. Van Proeyen, Symmetry structure of special geometries, Nucl. Phys. B 400 (1993) 463 [hep-th/9210068] [SPIRES].

[23] S. Bellucci, S. Ferrara, M. Günaydin and A. Marrani, Charge orbits of symmetric special geometries and attractors, Int. J. Mod. Phys. A 21 (2006) 5043 [hep-th/0606209] [SPIRES].

[24] E.G. Gimon, F. Larsen and J. Simon, Constituent model of extremal non-BPS black holes, JHEP 07 (2009) 052 [arXiv: 0903.0719] [SPIRES].

[25] A. Castro and J. Simon, Deconstructing the D0-D6 system, JHEP 05 (2009) 078 [arXiv: 0903.5523] [SPIRES].

[26] M. Günaydin, Lectures on spectrum generating symmetries and U-duality in supergravity, extremal black holes, quantum attractors and harmonic superspace, arXiv:0908.0374 [SPIRES].

[27] J.C. Baez, The octonions, math/0105155.

[28] S. Ferrara, E.G. Gimon and R. Kallosh, Magic supergravities, $N=8$ and black hole composites, Phys. Rev. D 74 (2006) 125018 [hep-th/0606211] [SPIRES]. 
[29] L. Andrianopoli, R. D'Auria, S. Ferrara, P.A. Grassi and M. Trigiante, Exceptional $N=6$ and $N=2 A d S_{4}$ supergravity and zero-center modules, JHEP 04 (2009) 074 [arXiv:0810.1214] [SPIRES].

[30] L. Andrianopoli, R. D'Auria and S. Ferrara, U-invariants, black-hole entropy and fixed scalars, Phys. Lett. B 403 (1997) 12 [hep-th/9703156] [SPIRES].

[31] D. Roest and H. Samtleben, Twin supergravities, Class. Quant. Grav. 26 (2009) 155001 [arXiv: 0904.1344] [SPIRES].

[32] R. Slansky, Group theory for unified model building, Phys. Rept. 79 (1981) 1 [SPIRES].

[33] B. de Wit, H. Samtleben and M. Trigiante, On lagrangians and gaugings of maximal supergravities, Nucl. Phys. B 655 (2003) 93 [hep-th/0212239] [SPIRES].

[34] A. Marrani, E. Orazi and F. Riccioni, Exceptional reductions, J. Phys. A 44 (2011) 155207 [arXiv: 1012.5797] [SPIRES].

[35] D. Gaiotto, W. Li and M. Padi, Non-supersymmetric attractor flow in symmetric spaces, JHEP 12 (2007) 093 [arXiv:0710.1638] [SPIRES].

[36] L. Borsten, D. Dahanayake, M.J. Duff and W. Rubens, Black holes admitting a Freudenthal dual, Phys. Rev. D 80 (2009) 026003 [arXiv:0903.5517] [SPIRES].

[37] S. Ferrara and A. Marrani, Matrix norms, BPS bounds and marginal stability in $N=8$ supergravity, JHEP 12 (2010) 038 [arXiv: 1009.3251] [SPIRES].

[38] G. Bossard, 1/8 BPS black hole composites, arXiv:1001.3157 [SPIRES].

[39] S. Ferrara and M. Günaydin, Orbits of exceptional groups, duality and BPS states in string theory, Int. J. Mod. Phys. A 13 (1998) 2075 [hep-th/9708025] [SPIRES].

[40] M. Cvetič and D. Youm, Dyonic BPS saturated black holes of heterotic string on a six torus, Phys. Rev. D 53 (1996) 584 [hep-th/9507090] [SPIRES].

[41] M. Cvetič and A.A. Tseytlin, General class of BPS saturated dyonic black holes as exact superstring solutions, Phys. Lett. B 366 (1996) 95 [hep-th/9510097] [SPIRES].

[42] M. Cvetič and A.A. Tseytlin, Solitonic strings and BPS saturated dyonic black holes, Phys. Rev. D 53 (1996) 5619 [Erratum ibid. D 55 (1997) 3907] [hep-th/9512031] [SPIRES].

[43] M.J. Duff, J.T. Liu and J. Rahmfeld, Four-dimensional string-string-string triality, Nucl. Phys. B 459 (1996) 125 [hep-th/9508094] [SPIRES]. 